\title{
Analysis of the pathogenic bacteria, drug resistance, and risk factors of postoperative infection in patients with non-small cell lung cancer
}

\author{
Yan Chen ${ }^{1,2 \#}$, Fangyuan Wen ${ }^{2,3 \#}$, Huacui Chen ${ }^{4}$, Ying Zhao ${ }^{1,2}$, Lei Ding ${ }^{2,5}$, Weifeng Lu ${ }^{2,6}$, Yu Liu ${ }^{2,7}$, \\ Yang Xue $\mathrm{Xu}^{2,8}$
}

${ }^{1}$ Pediatric Hematological Neoplasms, Sichuan Provincial People's Hospital, University of Electronic Science and Technology of China, Chengdu, China; ${ }^{2}$ Chinese Academy of Sciences, Sichuan Translational Medicine Research Hospital, Chengdu, China; ${ }^{3}$ Outpatient Department, Sichuan Provincial People's Hospital, University of Electronic Science and Technology of China, Chengdu, China; ${ }^{4}$ The Fifth Outpatients Department, The General Hospital of Western Theater Command Pal, Chengdu, China; ${ }^{5}$ Emergency Department, Sichuan Provincial People's Hospital, University of Electronic Science and Technology of China, Chengdu, China; ${ }^{6}$ Department of Anesthesia Operation Center, Sichuan Provincial People's Hospital, University of Electronic Science and Technology of China, Chengdu, China; ${ }^{7}$ Department of Oncology, Sichuan Provincial People's Hospital, University of Electronic Science and Technology of China, Chengdu, China; ${ }^{8}$ Department of Thoracic Surgery, Sichuan Provincial People's Hospital, University of Electronic Science and Technology of China, Chengdu, China

Contributions: (I) Conception and design: Y Chen, F Wen, H Chen; (II) Administrative support: F Wen, Y Zhao, L Ding; (III) Provision of study materials or patients: Y Chen, H Chen, Y Zhao, L Ding; (IV) Collection and assembly of data: W Lu, Y Liu, Y Xue; (V) Data analysis and interpretation: Y Chen, F Wen, H Chen, Y Liu, Y Xue; (VI) Manuscript writing: All authors; (VII) Final approval of manuscript: All authors.

\#These authors contributed equally to this work.

Correspondence to: Yu Liu. Department of Oncology, No.32 West Section 2, First Ring Road, Chengdu 610072, China. Email: ly14067599@163.com; Yang Xue. Department of Thoracic surgery, No.32 West Section 2, First Ring Road, Chengdu 610072, China. Email: 1659815999@qq.com.

Background: To analyze the pathogenic bacteria, drug resistance, and risk factors of postoperative infection in patients with non-small cell lung cancer (NSCLC).

Methods: A total of 119 patients with NSCLC who were admitted to our hospital from January 2017 to March 2020 were selected. The patients' clinical data were collected to evaluate the postoperative infection. The pathogenic bacteria, drug resistance, and risk factors of postoperative infections in patients with NSCLC were analyzed.

Results: Among 119 patients, 33 cases (27.73\%) had postoperative infection, and 86 cases (72.28\%) had no infection. In total, 81 pathogens were isolated from the secretions via bacterial culture from the infected sites of the 33 patients. Of these, 43 (53.09\%) were gram-negative bacteria, 34 (41.98\%) were Grampositive bacteria, and four (4.94\%) were fungi. Postoperative gram-negative infection showed the highest resistance rate to ciprofloxacin $(81.39 \%)$, and the drug resistance rate to imipenem and meropenem was low $(9.30 \%$ and $4.65 \%$, respectively). Postoperative gram-positive infection exhibited the highest resistance rate to erythromycin $(82.35 \%)$, and the drug resistance rate to vancomycin was low (5.88\%). According to the univariate analysis, there were differences between the two groups in age, length of hospitalization, combined diseases, operation time, invasive procedures, hemoglobin, and serum albumin $(\mathrm{P}<0.05)$. However, there were no differences in terms of gender, TNM staging, and pathological classification $(\mathrm{P}<0.05)$. Based on the unconditional multivariate logistic regression model analysis, age $\geq 60$ years, hospitalization time $>30 \mathrm{~d}$, combined diseases, operation time $\geq 3 \mathrm{~h}$, hospitalization time $>30 \mathrm{~d}$, invasive operation, hemoglobin $\leq 90 \mathrm{~g} / \mathrm{L}$, and serum albumin $\leq 30 \mathrm{~g} / \mathrm{L}$ were independent risk factors leading to postoperative infection in patients with NSCLC $(\mathrm{P}<0.05)$.

Conclusions: The postoperative infection rate of patients with NSCLC is high. gram-negative bacteria infection is the primary infection in patients. There are many factors that cause postoperative infections in patients, and it is necessary to strictly control these risk factors in clinical practice, which is an effective means to prevent postoperative infection. 


\begin{abstract}
Keywords: Non-small cell lung cancer (NSCLC); postoperative infection; pathogenic bacteria; drug resistance; risk factors
\end{abstract}

Submitted Aug 07, 2021. Accepted for publication Sep 10, 2021.

doi: 10.21037/apm-21-2364

View this article at: https://dx.doi.org/10.21037/apm-21-2364

\section{Introduction}

According to relevant reports, lung cancer is the leading cause of cancer-related mortality worldwide, and the incidence of lung cancer in China is high. In recent decades, the incidence of lung cancer has continued to rise, and the mortality and incidence of malignant tumors are ranked first (1). Approximately $80 \%$ of primary bronchial lung cancers are non-small cell lung cancer (NSCLC), with the rest being small cell lung cancer. At present, surgical treatment is still the main treatment for small cell lung cancer. However, these patients often have basic diseases, and the body is in a state of consumption during the treatment process, which leads to a further decline in the patient's resistance, and is more easily combined with infections, which are more common in lung infections (2). Understanding the postoperative complications of NSCLC patients and analyzing its characteristics and related factors are effective means to improve the therapeutic effect and prognosis of patients. At present, there are few studies on the distribution of pathogens and related drug resistance in NSCLC. Based on this, we collected the clinical data of NSCLC patients treated in our hospital, analyzed their infections and the distribution of pathogens, and understood the risk factors affecting the infection of patients, so as to provide a basis for reducing and preventing hospital infections. The report is as follows. We present the following article in accordance with the STROBE reporting checklist (available at https://dx.doi.org/10.21037/apm-21-2364).

\section{Methods}

\section{General information}

A total of 119 patients with NSCLC who were admitted to our hospital from January 2017 to March 2020 were selected. The inclusion criteria were as follows: (I) the patients' family members were aware of and agreed to this study, and signed the relevant informed consent; (II) patients with complete clinical data; and (III) patients diagnosed with NSCLC by clinical manifestations and imaging examinations. Patients were excluded based on the following criteria: (I) patients with other tumors; (II) patients with infection before treatment; (III) patients who were lost to follow-up; and (IV) patients with mental illnesses, such as depressive disorder and schizophrenia. The study was conducted in accordance with the Declaration of Helsinki (as revised in 2013). The study was approved by medical ethics committee of Sichuan Provincial People's Hospital [Lunshen (Research) No. 390, 2020]. The patients' family members were aware of and agreed to this study, and signed the relevant informed consent.

\section{Data collection}

The clinical data of patients was collected, including age, gender, length of hospital stay, comorbid diseases (hypertension, diabetes, hyperlipidemia, etc.), TNM staging (stage I-II, stage III), pathological classification (adenocarcinoma, squamous cell carcinoma), surgery time ( $\geq 3 \mathrm{~h},<3 \mathrm{~h}$ ), hospital stay ( $\geq 30 \mathrm{~d},<30 \mathrm{~d}$ ), invasive procedures, hemoglobin, serum albumin, etc.

\section{Grouping}

The diagnostic criteria for infection were as follows: (I) all patients underwent surgical treatment; (II) no infection occurred preoperatively; and (III) all patients were infected postoperatively. According to the relevant standards in the Diagnostic Standards for Nosocomial Infections, the diagnosis was confirmed by sputum bacterial culture, clinical manifestations, CT, and other examinations. The patients were divided into infected and uninfected groups according to whether they had a postoperative infection (3).

\section{Identification of pathogenic bacteria and drug resistance detection}

The specimens were collected from the infected sites of patients who had postoperative infection. Strict aseptic 
Table 1 Distribution of infectious pathogens after surgery

\begin{tabular}{lcc}
\hline Pathogenic bacteria & Strains & Rate (\%) \\
\hline Gram-positive bacteria & 43 & 53.09 \\
Staphylococcus aureus & 18 & 22.22 \\
Coagulase-negative Glucobacteria & 14 & 17.28 \\
Staphylococcus epidermidis & 7 & 8.64 \\
Other & 4 & 4.94 \\
Gram-negative bacteria & 34 & 41.98 \\
Pseudomonas aeruginosa & 15 & 18.52 \\
Klebsiella pneumoniae & 9 & 11.11 \\
Escherichia coli & 4 & 4.94 \\
Acinetobacter baumannii & 3 & 3.70 \\
Other & 3 & 3.70 \\
Fungus & 4 & 4.94 \\
Total & 81 & 100.00 \\
\hline
\end{tabular}

Table 2 Analysis of drug resistance of gram-negative bacteria after postoperative infection

\begin{tabular}{lcc}
\hline \multirow{2}{*}{ Antibacterial agents } & \multicolumn{2}{c}{ Gram-negative bacteria $(\mathrm{n}=43)$} \\
\cline { 2 - 3 } Ciprofloxacin & Strains & Drug resistance rate $(\%)$ \\
Ceftazidime & 35 & 81.39 \\
Ceftriaxone & 24 & 55.81 \\
Levofloxacin & 20 & 46.51 \\
Macrodantin & 19 & 44.18 \\
Imipenem & 8 & 18.60 \\
Meropenem & 4 & 9.30 \\
\hline
\end{tabular}

operation was carried out during specimen collection. Following collection, the specimens were sent for inspection, and an VITEK 2 Compact automatic microbial identification system (Bio Mérieux, Shanghai, China) was used for detection to analyze the distribution of pathogenic bacteria. For drug resistance, the minimum inhibitory concentration was used for drug susceptibility experiments (4).

\section{Observation indicators}

The pathogenic bacteria and drug resistance of postoperatively infected NSCLC patients were analyzed. The risk factors leading to postoperative infection were analyzed.

\section{Statistical methods}

The data in this study were statistically analyzed by SPSS22.0 software (SPSS, Chicago, Illinois, USA). Measurement data were described by the mean \pm standard deviation $(\overline{x \pm s})$, and the t-test was used. Counting data were described by pass rate or composition ratio, and $\chi^{2}$ test was used. Multivariate logistic regression analysis was used to analyze the risk factors of postoperative infection in patients with NSCLC. The difference was statistically significant when $\mathrm{P}<0.05$.

\section{Results}

\section{Postoperative infection of 119 patients}

Among the 119 included patients, 33 patients (27.73\%) had postoperative infections, and 86 patients $(72.28 \%)$ had no infections.

\section{Distribution of infectious pathogens after surgery}

The secretions from the infected sites of the 33 infected patients were cultured and treated, and 81 strains pathogenic bacteria were isolated. Of these, 43 strains (53.09\%) were gram-negative bacteria, 34 strains (41.98\%) were gram-positive bacteria, and four strains (4.94\%) were fungi. The specific distribution is shown in Table 1.

\section{Analysis of drug resistance of infectious pathogens after} surgery

Analysis of drug resistance of gram-negative bacteria after postoperative infection

As shown in Table 2, the highest rate of resistance to ciprofloxacin in gram-negative bacteria after surgery was $81.39 \%$, and the lowest rates of resistance to drugresistant imipenem and meropenem were $9.30 \%$ and $4.65 \%$, respectively.

Analysis of drug resistance of gram-positive bacteria after postoperative infection

As shown in Table 3, the highest rate of resistance to erythromycin after postoperative infection of gram-positive 
Table 3 Analysis of drug resistance of Gram-positive bacteria after postoperative infection

\begin{tabular}{lcc}
\hline \multirow{2}{*}{ Antibacterial agents } & \multicolumn{2}{c}{ Gram-positive bacteria $(\mathrm{n}=34)$} \\
\cline { 2 - 3 } & Strains & Drug resistance rate (\%) \\
\hline Erythrocin & 28 & 82.35 \\
Penicillin G & 19 & 55.88 \\
Levofloxacin & 16 & 47.59 \\
Gentamicin & 8 & 23.53 \\
Cefoxitin & 4 & 11.76 \\
Vancomycin & 2 & 5.88 \\
\hline
\end{tabular}

bacteria was $82.35 \%$, and the lowest rate of resistance to vancomycin was $5.88 \%$.

\section{Single-factor analysis of postoperative infection in patients with NSCLC}

According to the univariate analysis, there were differences between the two groups in terms of age, hospital stay, comorbid diseases, operation time, invasive procedures, hemoglobin, and serum albumin $(\mathrm{P}<0.05)$. However, there were no differences between the two groups in terms of gender, TNM staging, and pathological classification $(\mathrm{P}<0.05)$, as shown in Table 4.

\section{Analysis of multiple factors leading to postoperative infection in patients with NSCLC}

The significance and assignment of the factors to be analyzed are shown in Table 5. The unconditional multivariate logistic regression model analysis showed that age $\geq 60$ years old, hospitalization time $>30$ days, comorbid diseases, operation time $\geq 3$ hours, hospitalization time $>30$ days, invasive operation, hemoglobin $\leq 90 \mathrm{~g} / \mathrm{L}$, and serum albumin $\leq 30 \mathrm{~g} / \mathrm{L}$ were independent risk factors leading to postoperative infection in patients with NSCLC $(\mathrm{P}<0.05)$, as shown in Table 6.

\section{Discussion}

Most patients with NSCLC are at an advanced stage when first diagnosed, with a high malignancy stage and poor chemotherapy sensitivity, and thus, early treatment is crucial (5). Understanding the postoperative infection status can help to improve the prognosis and treatment effect of patients. In this study, 33 of the 119 included patients $(27.73 \%)$ developed postoperative infections. Through bacterial culture and drug susceptibility test analysis, it was found that gram-negative bacteria infections accounted for the majority, indicating that the pathogenic bacteria of patients with infection were invasive. The high resistance rate to erythromycin indicates that in clinical practice, appropriate antibiotics should be selected for treatment according to the drug sensitivity results after postoperative infection in NSCLC patients to avoid the abuse of antibiotics and reduce the occurrence of drug resistance (6).

The unconditional multivariate logistic regression model analysis showed that age $\geq 60$ years old, hospitalization time $>30$ days, comorbid diseases, operation time $\geq 3$ hours, invasive operation, hemoglobin $\leq 90 \mathrm{~g} / \mathrm{L}$, and serum albumin $\leq 30 \mathrm{~g} / \mathrm{L}$ were independent risk factors leading to postoperative infection in patients with NSCLC. We speculate that this may be caused by the following factors. Firstly, the relationship between postoperative infection and age could be due to the declining function of body tissues and organs in elderly patients, as well as the declining defense ability of the bronchial mucosa of such patients, which can cause respiratory tract infection in patients. At the same time, long-term hospitalization leads to a poor anti-infection ability against pathogens in the hospital. Numerous studies have shown that age is a risk factor that results in susceptibility to infection after surgical treatment $(7,8)$. Secondly, the relationship between postoperative infection and length of hospitalization could be attributable to the fact that patients with a longer hospitalization time have more serious illnesses, are in contact with other patients during hospitalization, and undergo an increased number of invasive operations, which can easily lead to nosocomial infection (9). Thirdly, the relationship between postoperative infection and comorbid diseases could be due to the fact that patients with underlying diseases typically have lower physical fitness and are more likely to develop infections after surgical treatment. Fourthly, the relationship between postoperative infection and operation time could be attributable to the fact that patients often choose the lateral position during surgical treatment, and intraoperative secretion retention is prone to occur in this position. Studies have shown that with the prolongation of operation time, secretion retention will increase significantly (10). Previous studies have reported that the time of anesthesia also increases after the operation time is extended. After the one-lung ventilation time increases, the time of pulmonary fistula will also 
Table 4 Single-factor analysis of postoperative infection in patients with non-small lung cancer cell (NSCLC)

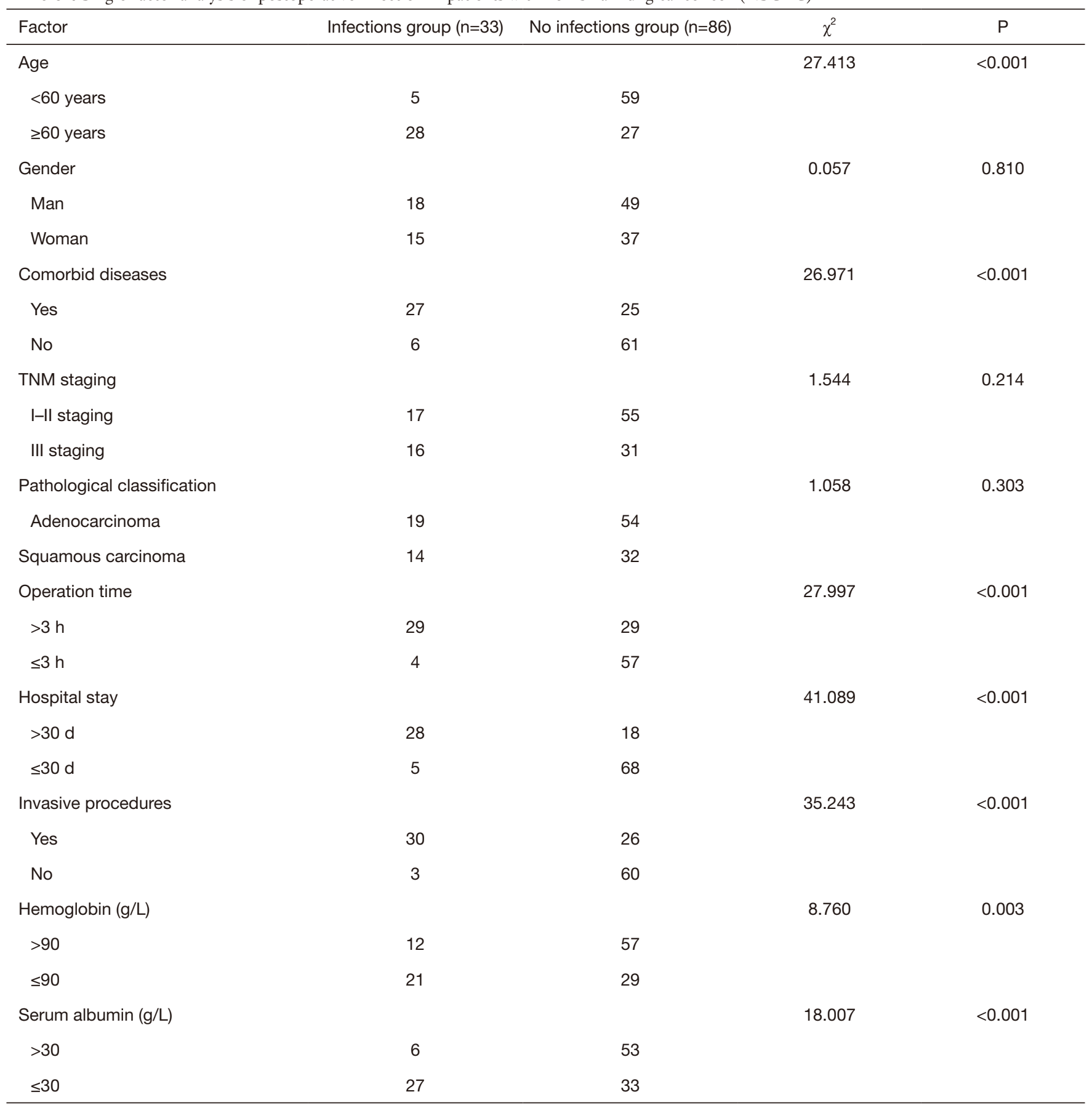

increase, leading to lung damage (11). However, patients may become irritable and restless after prolonged anesthesia time, which may cause respiratory function to be inhibited after taking sedatives. Thus, the patient may have difficulty in expectoration, a weakened cough emission, and is prone to pulmonary infection. Patients may have respiratory dysfunction, which can also lead to postoperative respiratory failure $(12,13)$. Chen et al. (14) reported that as the operation time is prolonged, the time of repeated rubbing and squeezing of the lungs is also increased, as is bronchial secretions, which significantly increases the postoperative lung infection rate (15). Fifthly, the relationship between 
Table 5 Significance and assignment of factors to be analyzed

\begin{tabular}{lll}
\hline Factors & Significance & Assignment \\
\hline X1 & Age & $0=<60$ years; $1=\geq 60$ years \\
X2 & Hospital stay & $0=\leq 30 \mathrm{~d} ; 1=>30 \mathrm{~d}$ \\
X3 & Comorbid diseases & $0=$ no; $1=$ yes \\
X4 & Operation time & $0=<3 \mathrm{~h} ; 1=\geq 3 \mathrm{~h}$ \\
X5 & Invasive procedures & $0=$ no; $1=$ yes \\
X6 & Hemoglobin & $0=>90 \mathrm{~g} / \mathrm{L} ; 1=\leq 90 \mathrm{~g} / \mathrm{L}$ \\
X7 & Serum albumin & $0=>30 \mathrm{~g} / \mathrm{L} ; 1=\leq 30 \mathrm{~g} / \mathrm{L}$ \\
\hline
\end{tabular}

Table 6 Multi-factor analysis of postoperative infection in patients with NSCLC

\begin{tabular}{lccccc}
\hline Factors & Regression coefficient & Standard error & Wald $\chi^{2}$ & P & OR (95\% Cl) \\
\hline X1=1 (Reference $=0)$ & 0.915 & 0.205 & 8.459 & $<0.001$ & $2.496(1.670-3.731)$ \\
X2=1 (Reference =0) & 0.889 & 0.316 & 9.184 & $<0.001$ & $2.432(1.309-4.519)$ \\
X3=1 (Reference =0) & 0.649 & 0.311 & 3.526 & 0.002 & $1.913(1.000-3.661)$ \\
X4=1 (Reference =0) & 0.711 & 0.334 & 3.157 & 0.018 & $2.036(1.057-3.918)$ \\
X5=1 (Reference =0) & 0.619 & 0.284 & 4.595 & 0.001 & $1.913(1.096-3.338)$ \\
X6=1 (Reference =0) & 0.705 & 0.236 & 8.715 & $<0.01$ & $2.024(1.274-3.214)$ \\
X7=1 (Reference =0) & 0.613 & 0.228 & 9.514 & $<0.01$ & $1.846(1.181-2.886)$ \\
\hline
\end{tabular}

postoperative infection and invasive operation could be due to the fact that endotracheal intubation is often required after surgery. This is an invasive operation that could easily damage the oral mucosa of the patient and destroy the balance of the oral flora. Studies have also shown that long-term pure oxygen inhalation can cause normal lung tissue damage and increase the risk of respiratory and pulmonary infections $(16,17)$. Lastly, the relationship between postoperative infection and hemoglobin/serum albumin could be attributable to the fact that patients with low levels of hemoglobin and serum albumin levels have a poor nutritional status. Thus, the body cannot provide the consumed capacity, and the body's impaired immunity will lead to infection (18).

From the above analysis of risk factors, it is clear that in order to reduce the risk of postoperative infection, clinical attention needs to be paid to patients who exhibit these risk factors. Older patients need to strengthen nutrition before surgery to improve their immunity. Doctors should also try to avoid prolonging the operation time and reduce surgical trauma. Furthermore, invasive operations after surgery should be reduced. During surgical treatment, doctors should avoid invasive operations and act gently. When the patient's condition permits, the use of a ventilator should be reduced, and it is best to perform sputum suction in the operating room. The correct use of antibiotics during treatment. If the tube can be taken offline as soon as possible, the incidence of nosocomial infection can be reduced (19).

In summary, the postoperative infection rate of patients with NSCLC is high, and the main cases are gram-negative infections. There are numerous postoperative infection factors in patients, and these need to be strictly controlled in clinical practice, which is an effective means to prevent postoperative infection. However, the number of people included in this study is limited, and the follow-up sample size can be increased to expand the research scope and increase the research direction.

\section{Acknowledgments}

Funding: None. 


\section{Footnote}

Reporting Checklist: The authors have completed the STROBE reporting checklist. Available at https://dx.doi. org/10.21037/apm-21-2364

Data Sharing Statement: Available at https://dx.doi. org/10.21037/apm-21-2364

Conflicts of Interest: All authors have completed the ICMJE uniform disclosure form (available at https://dx.doi. org/10.21037/apm-21-2364). The authors have no conflicts of interest to declare.

Ethical Statement: The authors are accountable for all aspects of the work in ensuring that questions related to the accuracy or integrity of any part of the work are appropriately investigated and resolved. The study was conducted in accordance with the Declaration of Helsinki (as revised in 2013). The study was approved by medical ethics committee of Sichuan Provincial People's Hospital [Lunshen (Research) No. 390, 2020]. The patients' family members were aware of and agreed to this study, and signed the relevant informed consent.

Open Access Statement: This is an Open Access article distributed in accordance with the Creative Commons Attribution-NonCommercial-NoDerivs 4.0 International License (CC BY-NC-ND 4.0), which permits the noncommercial replication and distribution of the article with the strict proviso that no changes or edits are made and the original work is properly cited (including links to both the formal publication through the relevant DOI and the license). See: https://creativecommons.org/licenses/by-nc-nd/4.0/.

\section{References}

1. Zheng XQ, Huang JF, Lin JL, et al. Incidence, prognostic factors, and a nomogram of lung cancer with bone metastasis at initial diagnosis: a population-based study. Transl Lung Cancer Res 2019;8:367-79.

2. Palade E, Günter J, Gomez JMM, et al. Morbidity, mortality and long-term outcome of lung cancer resections performed in palliative intent. J Thorac Dis 2019;11:4308-18.

3. Ferrell CL, Barnhart MD, Herman E. Impact of postoperative antibiotics on rates of infection and implant removal after tibial tuberosity advancement in 1,768 canine stifles. Vet Surg 2019;48:694-9.

4. Qin L, Qi ZF. Efficacy of therapy for non-small cell lung cancer acquired epidermal growth factor receptor tyrosine kinase inhibitor resistance. Chinese Journal of Clinical Oncology and Rehabilitation 2019;26:291-93.

5. Tu $\mathrm{H}, \mathrm{Wu} M$, Huang $\mathrm{W}$, et al. Screening of potential biomarkers and their predictive value in early stage nonsmall cell lung cancer: a bioinformatics analysis. Transl Lung Cancer Res 2019;8:797-807.

6. Gao YF, Mao LH, Jin AH. Effect of traditional Chinese medicine syndrome differentiation and nursing on quality of life and immune function of elderly patients with middle and advanced non-small cell lung cancer. Chinese Journal of Modern Nursing 2021;27:1637-41.

7. Ye XM, Zhu WH, Huang LJ, et al. Drug resistance genes in extended spectrum $\beta$-lactamases-producing pathogens isolated from non-small cell lung cancer patients with pulmonary infection during chemotherapy. Chinese Journal of Hospital Infection 2019;29:166-70.

8. Qiu P, Zheng X, Tan XB, et al. Related factors influencing the recurrence and metastasis of non-small cell lung cancer after minimally invasive resection. Practical Journal of Cancer 2020;35:598-602.

9. Mamesaya N, Narita SN, Naito T, et al. 1507P Nivolumab-induced and radiation recall pneumonitis in patients with non-small cell lung cancer: A multicenter real world analysis of 669 patients. Ann Oncol 2019;30:618.

10. Zhang Q, Liu SX, Wang LG, et al. Relationship between postoperative pulmonary infection and pathogenic bacteria colonized in the airway of patients with non-small cell lung cancer. Medical \& Pharmaceutical Journal of Chinese People's Liberation Army 2020;32:13-17.

11. Zhang Z, Li L, Yang Q, et al. Efficacy and prognosis of radio-frequency ablation in patients with non-small cell lung cancer after EGFR-TKIs resistance. Journal of Clinical Pulmonary Medicine 2019;24:890-5.

12. Witlox WJA, Ramaekers BLT, Groen HJM, et al. Factors determining the effect of prophylactic cranial irradiation (PCI) in patients with stage-III nonsmall cell lung cancer: exploratory subgroup analyses of the NVALT-11/ DLCRG-02 phase-III study. Acta Oncol 2019;58:1528-31.

13. Cao K, Chen ZT, Chang L, et al. Progress research on cell-free DNA in non-small cell lung cancer. Journal of Cancer Control and Treatment 2019;32:849-57.

14. Chen SX, Chen Y, Zhang ZJ, et al. Distribution and influencing factors of nosocomial infection in patients with non-small cell lung cancer after operation. Journal of Clinical Pulmonology 2020;25:122-5. 
15. Schwalk AJ, Ost DE, Saltijeral SN, et al. Risk Factors for and Time to Recurrence of Symptomatic Malignant Pleural Effusion in Patients With Metastatic Non-Small Cell Lung Cancer with EGFR or ALK Mutations. Chest 2021;159:1256-64.

16. Jiang WC. Related factors of postoperative pathologically confirmed stage N1-2 lymph node metastasis in patients with clinical stage N0 NSCLC. Anhui Medical and Pharmaceutical Journal 2019;23:1373-75.

17. Xu S, Du B, Shan A, et al. The risk factors for the postoperative pulmonary infection in patients with hypertensive cerebral hemorrhage: A retrospective analysis. Medicine (Baltimore) 2020;99:e23544.

Cite this article as: Chen Y, Wen F, Chen H, Zhao Y, Ding L, Lu W, Liu Y, Xue Y. Analysis of the pathogenic bacteria, drug resistance, and risk factors of postoperative infection in patients with non-small cell lung cancer. Ann Palliat Med 2021;10(9):1000510012. doi: 10.21037/apm-21-2364
18. Guo Z, Zhang J, Gong Z, et al. Correlation of factors associated with postoperative infection in patients with malignant oral and maxillofacial tumours: a logistic regression analysis. Br J Oral Maxillofac Surg 2019;57:460-5.

19. Liu QH, Li ZH, Li DB. Progress on Acquired Drugresistance Mechanism of EGFR-TKIs and Its Relation with MicroRNAs in Patients with Non-small Cell Lung Cancer. Practical Journal of Cardiac Cerebral Pneumal and Vascular Disease 2019;27:9-14.

(English Language Editor: A. Kassem) 